\title{
EFFECT OF RF PHASE MODULATION NEAR A PARAMETRIC RESONANCE ON THE LONGITUDINAL EMITTANCE
}

\author{
F. Orsini, CEA SACLAY, Gif-sur-Yvette, France \\ A. Mosnier, Projet SOLEIL, Gif-sur-Yvette, France
}

\begin{abstract}
Aiming at increasing the apparent bunchlength and hence the beam life time in electrons storage rings, RF phase modulation near one parametric resonance has been experimentally investigated. Since the eventual benefit of this technique depends greatly on the ring parameters, we studied the effect of such a modulation for different RF parameters on the longitudinal emittance. Theoritical predictions and results of simulations are compared and discussed. It is shown that synchrotron radiation tends to spoil the parametric resonance. In particular, a criterion for islands survival has been found.
\end{abstract}

\section{INTRODUCTION}

In order to reach very high brilliance, Synchrotron Radiation Light Sources demand intense bunches with very small transverse and longitudinal emittances. However the high density of electrons increases the Touschek effect and thus reduces the beam lifetime. In order to reduce the electron density, different approaches have been considered: a higher harmonic cavity operating in the bunch lengthening mode or a RF phase modulation which increases the apparent bunchlength but also the energy spread of the beam. This paper focus on the second method near the thirdinteger resonance, because the integer resonance is not an appropriate solution: well developed in [1], it is briefly illustrated here with the example of SOLEIL storage ring with the main ring parameters (see Table 1). Main simulation results proove that the integer resonance is too strong (see figure 1) and leads to distinct bunchlets in phase space and induces a dipole oscillation of the whole bunch as far as the amplitude of the modulation is important, whatever the regime of the modulation frequency is. In order to have an appropriate phase space occupied by the beam, the thirdinteger resonance parameters, frequency $\omega_{m}$ and amplitude $A_{m}$, must be first properly chosen. The method of the optimization parameters is discussed in details with some theory and numerical results. Then the synchrotron radiation effect, is studied in opposition with the parametric resonance effect with help of a Fokker-Planck treatment, which leads to the islands formation criterion. Also, further examples are given to illustrate this criterion.

\section{THIRD-INTEGER RESONANCE}

\subsection{Fixed Points and Islands Width}

The complete perturbated Hamiltonian in $(\phi, \delta)$ variables is, with $\phi$ the phase and $\delta$ the energy deviation of a particle,

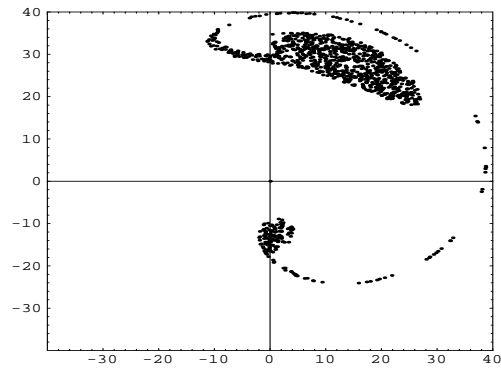

Figure 1: Particles in normalized phase space $(\phi, \delta)$ with modulation at the integer resonance in the regime just before the vanishing of the centered bunchlet.

$\overline{\phi_{s}}$ the synchronous angle (in convention $\overline{\phi_{s}}=\pi-\phi_{s}$ ) and $\omega_{s}$ the synchrotron frequency:

$$
\begin{aligned}
& H(\phi, \delta)=\frac{\omega_{s}}{2} \delta^{2}+\omega_{s} \tan \overline{\phi_{s}}\left(\sin \phi \cos \left(A_{m} \sin \omega_{m} t\right)\right. \\
& \left.+\cos \phi \sin \left(A_{m} \sin \omega_{m} t\right)\right)-\omega_{s} \cos \phi \cos \left(A_{m} \sin \omega_{m} t\right) \\
& +\omega_{s} \sin \phi \sin \left(A_{m} \sin \omega_{m} t\right)-\omega_{s} \phi \tan \overline{\phi_{s}}
\end{aligned}
$$

Using action-angle variables $(\tilde{J}, \tilde{\psi})$ in a rotating frame:

$\delta=-\sqrt{2 \tilde{J}} \cos \left(\tilde{\psi}+\omega_{m} t / 3\right), \phi=-\sqrt{2 \tilde{J}} \sin (\tilde{\psi}+$ $\left.\omega_{m} t / 3\right)$, expanding into Bessel functions and assuming to be close to the third-integer resonance, the Hamiltonian has the much simple form [1]:

$K(\tilde{J}, \tilde{\psi})=\left(\omega_{s}-\frac{\omega_{m}}{3}\right) \tilde{J}-\frac{\omega_{s} \tilde{J}^{2}}{16}-\frac{\omega_{s} A_{m}(2 \tilde{J})^{\frac{3}{2}}}{48} \cos 3 \tilde{\psi}$

In the new phase space $(\tilde{J}, \tilde{\psi})$, the stationary trajectories correspond to the H-constant contours. For appropriate modulation parameters, the 3 islands of the third-integer parametric resonance can be well-shaped. The coordinates of the stable fixed points (SFP: $\tilde{\psi}=0, \frac{2 \pi}{3}, \frac{4 \pi}{3}$ ) $\left(\frac{\partial K}{\partial \tilde{\psi}}=\frac{\partial K}{\partial \tilde{J}}=0\right)$ are:

$$
\begin{aligned}
& \phi_{\sigma}=0, \frac{\sqrt{3} a_{m}}{4}\left[1+R_{F P}\right], \frac{-\sqrt{3} a_{m}}{4}\left[1+R_{F P}\right] \\
& \delta_{\sigma}=\frac{a_{m}}{2}\left[1+R_{F P}\right], \frac{-a_{m}}{4}\left[1+R_{F P}\right], \frac{-a_{m}}{4}\left[1+R_{F P}\right]
\end{aligned}
$$

with the factor $R_{F P}=\sqrt{1+\frac{64 Q_{s}^{2}}{a_{m}^{2}\left(\sigma_{\epsilon} h \alpha\right)^{2}} \cdot\left(1-\frac{\omega_{m}}{3 \omega_{s}}\right)}$, where $a_{m}$ is the normalized modulation amplitude ( $A_{m}$ is in units of rms bunchlength), $Q_{s}$ is the synchrotron tune, $\sigma_{\epsilon}$ is the natural energy spread, $\alpha$ is the momentum compaction and $h$ is the harmonic number. The fixed points position depends on the ring and the RF modulation parameters. In order to depopulate the bunch center as much 
as possible, islands have to be placed close to the bunch core and to be large enough. However, eq. 3 shows that the SFPs can never reach the origin, even for a vanishing distance to the resonance $\left(\omega_{m}-3 \omega_{s}\right)$ and are bounded by a lower limit.

The island width calculation completes the islands structure. The island width is given by the distance between the separatrice, i.e the curve which joins the unstable fixed points, and the SFPs, where the hamiltonian is maximum [2]. The normalized island width (in $\sigma$ units) and expressed with storage ring parameters (eq. 4) is given by:

$$
\Delta \delta_{\sigma}= \pm 16 \sqrt{\frac{2}{3}}\left(\frac{Q_{s}}{\alpha \sigma_{\epsilon} h}\right)^{\frac{3}{2}}\left(1-\frac{\omega_{m}}{3 \omega_{s}}\right)^{\frac{3}{4}} \frac{1}{\sqrt{a_{m}} R_{F P}}
$$

This equation reveals that the more $\omega_{m}$ tends to $3 \omega_{s}$, the more the island width is reduced $\left(\Delta \delta_{\sigma} \propto\left(1-\frac{\omega_{m}}{3 \omega_{s}}\right)^{1 / 4}\right)$. There is then a trade-off between island position $\left(\omega_{m}\right.$ very close to $\left.3 \omega_{s}\right)$ and the island width $\left(\omega_{m}\right.$ not too close to $\left.3 \omega_{s}\right)$.

\subsection{RF phase modulation optimization}

Different Synchrotron Light Sources were studied [3]. Table 1 resumes the relevant parameters for Soleil, Bessy I and SuperAco.

Table 1: Synchrotron Light Sources main RF parameters

\begin{tabular}{|l|c|c|c|}
\hline & Soleil & Bessy I & SuperAco \\
\hline$F_{R F}(\mathrm{MHz})$ & 352.2 & 499.2 & 100.0 \\
$h$ & 396 & 104 & 24 \\
$\alpha$ & $4.7710^{-4}$ & $1.510^{-2}$ & $1.4810^{-2}$ \\
$T_{\text {rad }}(\mathrm{ms})$ & 4.33 & 10.0 & 8.5 \\
$\sigma_{\epsilon}$ & $9.2410^{-4}$ & $5.010^{-4}$ & $5.510^{-4}$ \\
\hline
\end{tabular}

Results of optimization are given in table 2. Both parameters, amplitude and frequency of RF phase modulation, have to be first optimized in such a way that the SFPs are close to the bunch core, while keeping a sufficient island width and the stationary trajectories in phase space are shown in fig. 2 for one of the example. It is worth noting that $a_{m}$ must be not too large ( 1 or 2 maximum) for preventing any coherent movement of the whole bunch.

Table 2: Final optimization of the RF phase modulation parameters and islands characteristics

\begin{tabular}{|l|c|c|c|}
\hline & Soleil & Bessy I & SuperAco \\
\hline$\omega_{m} / \omega_{s}$ & 2.9995 & 2.9850 & 2.9950 \\
$A_{m}$ (degrees) & 1.48 & 5.68 & 3.24 \\
Island width $(\sigma)$ & 2.29 & 2.71 & 2.77 \\
\hline
\end{tabular}

When the bunch length is small (ex. Soleil), compared to the RF wave length, the modulation frequency has to be moved very close to $3 \omega_{s}$ in order to draw the SFPs to the

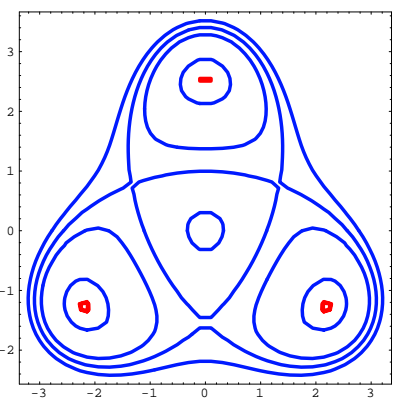

Figure 2: Soleil: separatrices in normalized phase space $\left(\phi_{\sigma}, \delta_{\sigma}\right)$.

origin. The islands width, table 2 , gives the maximum extension trajectory inside one island. For the three machines, particles, located at $1 \sigma$, will be drawn out up to nearly $3 \sigma$.

\section{SYNCHROTRON RADIATION EFFECT}

In this section, examples are given with a multi-particle tracking code [4] which simulates the movement of the particles of a bunch faced with RF phase modulation, synchrotron radiation and quantum excitation. The simulation is based on the recurrent equations for each particle:

- $\phi_{n+1}=\phi_{n}+2 \pi Q_{s} \times \delta_{n}$

- $\delta_{n+1}=\left(1-\frac{2}{T_{\text {rad }} F_{0}}\right) \cdot \delta_{n}+\frac{2}{\sqrt{T_{\text {rad }} F_{0}}} \sigma_{\epsilon} R_{I}$ $-\frac{Q_{s}}{\cos \overline{\phi_{s}}}\left(\sin \left(\overline{\phi_{s}}+\left(\phi_{n+1}+A_{m} \sin \omega_{m} t\right)\right)-\sin \overline{\phi_{s}}\right)$

$R_{I}$ is a random number used for the gaussian shape of the bunch.

\subsection{Fokker-Planck treatment}

In the previous analytical treatment, the synchrotron radiation effect, which includes the synchrotron radiation damping and the quantum excitation, has not been taken into account. Although, this effect has been neglected in previous papers, some strong effects have been observed in multiparticle tracking simulations. The previous Hamiltonian treatment can not be applied with the synchrotron radiation effect because of the non-conservative system properties. Our present analysis is based on the Fokker-Planck equation:

$$
\frac{\partial F}{\partial t}+\{H, F\}=R
$$

where $F(\phi, \delta, t)$ is the distribution function in the bunch, $R$ is the collision term describing the synchrotron radiation effect, $\{\cdots\}$ denotes the Poisson bracket and $H$ is our peturbated Hamiltonian previously explained.

Expressing eq. 5 in $(\tilde{J}, \tilde{\psi})$ variables and having now a system completely time-independant (details can be found in [4]), the final equation can be written as :

$$
\begin{aligned}
& {\left[\frac{\omega_{s} A_{m}(2 \tilde{J})^{3 / 2}}{32} \sin 3 \tilde{\psi} \tan ^{2} \tilde{\psi}+\gamma_{d} \tilde{J}+\kappa\right] \frac{\partial S}{\partial \tilde{J}}} \\
& +\tilde{J} \kappa \frac{\partial^{2} S}{\partial \tilde{J}^{2}}+\gamma_{d} S=0
\end{aligned}
$$


and the azimuthal periodicity for the third-integer resonance $F(\tilde{J}, \tilde{\psi})=S(\tilde{J}) \times e^{j 3 \tilde{\psi}}$ has been used. The quantity of interest is the term in bracket, which contains the third-integer resonance perturbation, the damping term with $\gamma_{d}$ and the quantum excitation term with $\kappa$ related to $\gamma_{d}$ by the relation $\sigma_{\epsilon}=\sqrt{\kappa / \gamma_{d}}$. The RF phase modulation will be still efficient if the magnitude of the first coefficient will be larger than the synchrotron radiation effect $\left(2^{n d}\right.$ and $3^{r d}$ coefficients).

\section{2 islands formation criterion}

Coming back with normalized phase space variables $(\phi, \delta)$, we express the three coefficients of the bracket term in eq. 6 as follows:

$c_{1}=\frac{\omega_{s} A_{m}}{16}\left(\frac{\alpha h \sigma_{\epsilon}}{Q_{s}}\right)^{3}, c_{2}=\frac{1}{T_{\text {rad }}}\left(\frac{\alpha h \sigma_{\epsilon}}{Q_{s}}\right)^{2}, c_{3}=\frac{1}{T_{\text {rad }}} \sigma_{\epsilon}^{2}$.

The $c_{3}$ term, much smaller than $c_{1}$ and $c_{2}$, can be neglected. We find a limit value on the energy spread for the formation of islands, whenever the beam energy spread will be smaller, islands are destroyed by the synchrotron radiation:

$$
\sigma_{\epsilon}>\sqrt{\frac{1}{T_{r a d}} \times \frac{16 Q_{s}}{\omega_{R F} \alpha^{2} h a_{m}}}
$$

\subsection{Simulations of formation or absence of is- lands}

We checked the validity of the island survival criterion for the 3 previously cited machines. For each machine, the RF phase modulation parameters $\left(\omega_{m}, a_{m}\right)$ have been first optimized to get well-shaped islands. Multi-particle simulations were performed for island formation or destruction with 2 values of energy spread: the natural one and a fictive one, which gives the reverse situation.

Figure 3 gives the particle distribution in phase space, showing the island destruction for Soleil (upper-left), SuperAco (bottom-left) and the island formation for Bessy I (middle-right) with their natural energy spread. Table 3 resumes the energy spread values, which were tested, as well as the limit value (natural energy spread are in bold characters). The energy spread of SuperAco, larger than the natural one and which was chosen for island creation, corresponds to a real situation, where the beam current is well above the turbulent regime.

Table 3: $\sigma_{\epsilon}$ parameter for island formation or absence.

\begin{tabular}{|l|c|c|c|}
\hline & Soleil & Bessy I & SuperAco \\
\hline$\sigma_{\text {elim }}$ & $11.1310^{-3}$ & $4.1410^{-4}$ & $14.0210^{-4}$ \\
\hline$\sigma_{\epsilon \text { island }}$ & $15.010^{-3}$ & $\mathbf{5 . 0 1 0} \mathbf{1 0}^{-4}$ & $18.3310^{-4}$ \\
\hline$\sigma_{\text {edamping }}$ & $\mathbf{9 . 2 4} \mathbf{1 0}^{-4}$ & $2.310^{-4}$ & $\mathbf{5 . 5 1 0} \mathbf{1 0}^{-4}$ \\
\hline
\end{tabular}
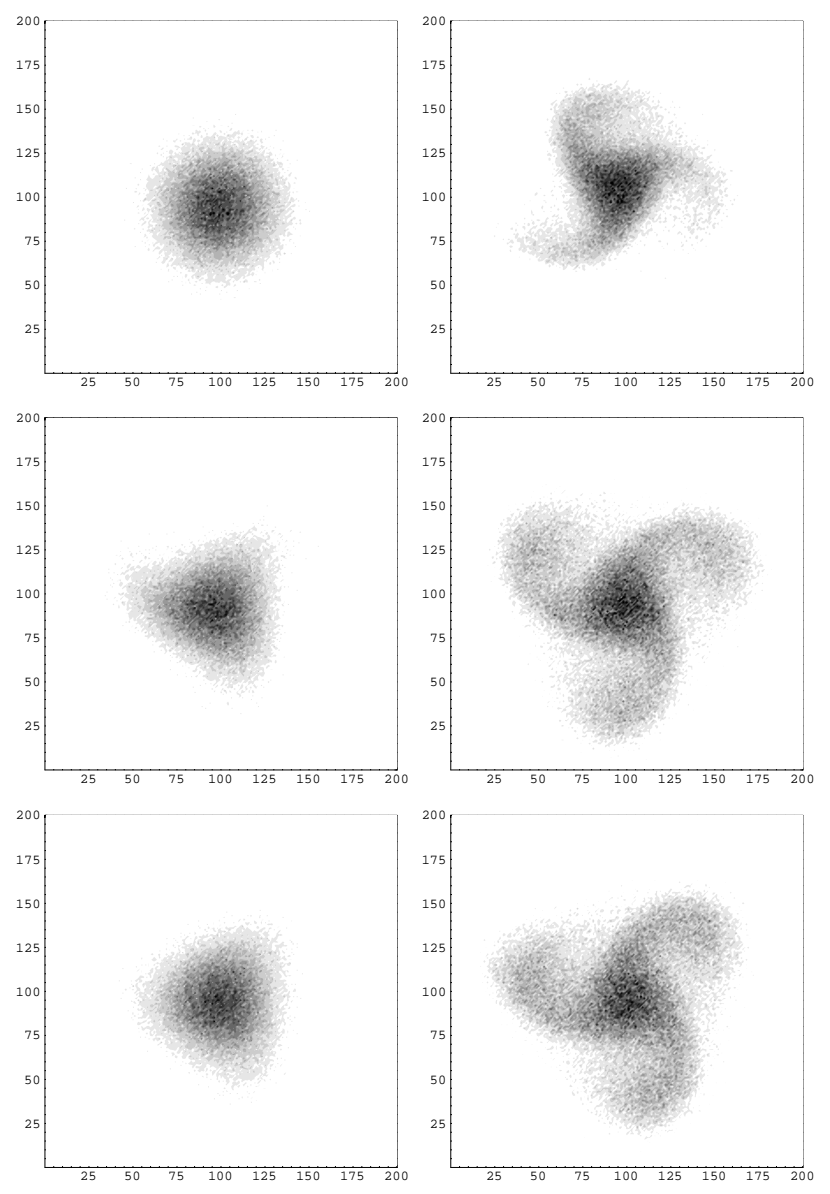

Figure 3: Snapshots ( $10^{5}$ particles) in normalized phase space $\left(\phi_{\sigma}, \delta_{\sigma}\right)$ with islands destruction (left) and islands formation (right) for, in order of appearance, Soleil, Bessy I and SuperAco.

\section{CONCLUSION}

With appropriate modulation parameters, the method can dilute the phase space. However, for some cases, synchrotron radiation effect prevents for the island formation and a criterion on the minimum required energy spread has been found. Anyway, when the $3 \omega_{s}$ excitation is successful, the energy spread is increased, by the same bunchlengthening factor (islands are rotating in phase space).

\section{REFERENCES}

[1] H. Huang and al.,"Experimental determination of the Hamiltonian for Synchrotron motion with rf phase modulation", Physical Review E, Vol.48, N6, Dec.1993.

[2] R.D. Ruth,'Single-particle dynamics and nonlinear resonances in circular accelerators", Lecture Notes in Physics, Vol.247, Springer Verlag, Proceedinds Sardinia 1985.

[3] P. Kuske, Yu. Senichev, MP. Level: private communications.

[4] F. Orsini A. Mosnier,' Is RF Phase Modulation a practical method for bunchlengthening ?", DAPNIA/SEA 99-03, March 1999. 